\title{
Minimum Field Requirements for Spin-Polarized Current Assisted Switching of Magnetization in Nanostructures with Uniaxial Anisotropy
}

\author{
Mihai DIMIAN ${ }^{1,2}$, Anca GINDULESCU ${ }^{1}$, Cyril ACHOLO ${ }^{2}$ \\ ${ }^{1}$ Department of Electrical Engineering and Computer Science, Stefan cel Mare University, \\ Suceava 720229, Romania \\ ${ }^{2}$ Department of Electrical and Computer Engineering, Howard University, \\ Washington, District of Columbia 20059, United States of America \\ dimian@eed.usv.ro
}

\begin{abstract}
The present paradigm of magnetic data storage is approaching its fundamental limits for areal storage density, as well as for speed in data processing. As a result, several magnetic recording alternatives, such as spin polarized current assisted recording, precessional switching, toggle switching, heat assisted recording are currently under intense research efforts. This article is aimed at providing a pertinent theoretical analysis of the spin polarized current assisted recording, emphasizing its performance with respect to minimum requirements for switching field. The first analytical derivation of the critical field curve in the presence of spin polarized currents is presented and the results are compared to the classical Stoner-Wohlfarth astroid. The analysis is performed under the framework of the Landau-LifshitzGilbert-Slonczewski equation for describing the magnetization dynamics driven by external magnetic fields and spin polarized currents.
\end{abstract}

Index Terms-Magnetic memory, Spintronics, LandauLifshitz equation, Bifurcation theory

\section{INTRODUCTION}

The evolution of magnetic data storage technology has been impressive since 1955, when IBM built the first hard disk drive featuring a storage capacity of 5MB with an areal recording density of $2 \mathrm{kbit} / \mathrm{in}^{2}$. The steady progress made in this area over the last fifty-three years has been driven by three figures of merit: the increase in storage density, the increase in speed of data processing, and the decrease in fabrication costs. The barrier of $100 \mathrm{Gbit} / \mathrm{in}^{2}$ has already been passed in 2002 [1,2] and demonstrations with area recording densities as high as $1 \mathrm{Tbit} / \mathrm{in}^{2}$ are expected in the near future based on perpendicular recording technology [3, 4]. It is apparent from these data that the storage of an information bit is related to nanometer scale magnetic thin film structures. Moreover, magnetic patterned media with a single-bit-per-island recording methodology have also been considered as recording media and have successfully passed preliminary tests $[5,6]$. In addition to the interest in the areal storage densities, a special emphasis is placed on the data rate of the hard disk drive. The current disk drives operate at a maximum internal data transfer rate of approximately $130 \mathrm{MB} / \mathrm{s}$, which corresponds to a channel data rate of $1.17 \mathrm{Gbit} / \mathrm{s}$ (using an 8/9 modulation code). Therefore, the writing time for a single bit, or equivalently magnetization reversal time, is below $1 \mathrm{~ns}$.
Another major research effort in the magnetic data storage has been recently devoted to magnetoresistive random access memory (MRAM), the first commercial version being produced by Freescale Semiconductors at the end of 2006. MRAM has the potential to store data at a high density, to access them with a high speed, and to be a low power consumer $[7,8]$. Once the performances in these directions become comparable with the ones of the semiconductors based memory, the nonvolatility property could determine the use of MRAM as a "universal memory". The most common design for MRAM uses a magnetic tunnel junction: two ferromagnetic thin films play the role of electrodes and a thin tunneling barrier separates them. The resistance of the tunneling junction is significantly modified as the magnetic moments of the ferromagnetic layers change their relative orientation. The difference in junction resistances corresponding to the stable parallel and anti-parallel orientations, respectively, makes possible the definition of the binary memory states. The ferromagnetic thin film electrodes have nanometer dimensions and the magnetization reversal time in these devices is in nanosecond regime.

In spite of the impressive progress over the years, the paradigm of magnetic data storage is now approaching its fundamental limits for areal storage density, as well as for speed in data processing $[9,10]$. Consequently, there is an urgent need for reliable alternatives to current magnetic recording techniques, which should feature sub-StonerWohlfarth reversal magnetic fields and sub-nanosecond magnetization reversal time. Several unconventional magnetic recording techniques, such as spin polarized current assisted recording [11, 12], precessional switching $[13,14]$, toggle switching $[15,16]$, heat assisted recording $[17,18]$ are currently under intense research efforts. While all previously mentioned techniques are characterized by sub-Stoner-Wohlfarth switching field, their implementations raise multiple challenges with respect to the design of applied magnetic field pulses and magnetization behavior. In conclusion, additional analyses are needed for understanding and controlling these new recording alternatives that promise to significantly improve the technical characteristics of magnetic data storage devices, such as HDD and MRAM. 


\section{MAGNETIZATION REVERSAL DRIVEN BY MAGNETIC FIELD AND SPIN POLARIZED CURRENT}

It has been proven theoretically $[19,20]$ and later experimentally [21] that the partially aligned spins of the electrons forming a spin polarized electric current have a much larger influence on the magnetization of a nanometer thin film than the classical magnetic field produced by that current. Thus, the interaction between the current moment and the nano-object magnetization can lead to magnetization oscillatory phenomena and magnetization reversals. These properties led to a growing interest in detailed analyses of magnetization dynamics driven by spin polarized currents and their possible applications in magnetic memories and microwave devices [22-26]. A large number of presentations given at the IEEE International Magnetics Conference, Madrid, May 3-8, 2008, have been dedicated to this research area, including the plenary lecture of Prof. Dr. Albert Fert, 2007 Nobel Laureate in Physics.

Our work is aimed at providing a pertinent theoretical analysis of the spin polarized current assisted recording, emphasizing its performance with respect to minimum requirements for switching field. An invited presentation on this work was given to the International Conference on Materials for Electrical Engineering, Bucharest, June 16-18, 2008.

The analysis is performed under the framework of Landau-Lifshitz-Gilbert-Slonczewski (LLGS) equation for describing the magnetization dynamics driven by external magnetic fields and spin polarized currents. Before proceeding to the analysis of this equation, we would like to mention several studies [12, 27-28] that presented numerical and analytical results related to our topic. They have given us the opportunity to better understand the spin-polarized current assisted reversal phenomena and have motivated us to start the analysis presented below. The main contribution of this article is the analytical derivation for the critical field curve in the presence of spin polarized current, coined as generalized Stoner-Wohlfarth astroid.

Consider a uniformly magnetized nano-object with uniaxial anisotropy subject to a spin polarized current and an external magnetic field, both spatially uniform. Since we focus on nanometer scale phenomena, it seems reasonable to consider spatially uniform quantities. Moreover, the exchange interaction is dominant at that scale and, consequently, the magnetic nano-objects can be considered uniformly magnetized regardless of their geometrical shape.

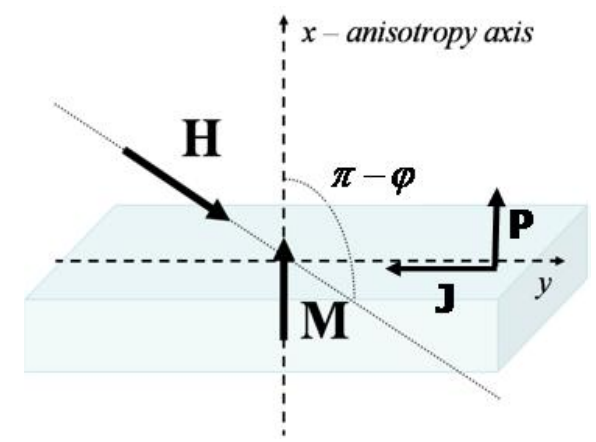

Figure 1. Configuration for spin polarized assisted magnetization reversal of a magnetic nano-cell in perpendicular thin film media subject to an external magnetic field applied at an angle $\pi-\varphi$ with the $\mathrm{x}$-axis (easy anisotropy axis)
The LLGS equation describing the magnetization dynamics driven by magnetic fields and spin polarized currents can be written in the following dimensionless form:

$$
\frac{d \mathbf{m}}{d t}-\alpha \mathbf{m} \times \frac{d \mathbf{m}}{d t}=-\mathbf{m} \times\left(\mathbf{h}_{e f f}-\beta \mathbf{m} \times \mathbf{p}\right),
$$

where $\mathbf{m}=\mathbf{M} / M_{s}, \mathbf{h}_{e f f}=\mathbf{H}_{e f f} / M_{s}$, while time $t$ is expressed in units of $\left(\gamma M_{s}\right)^{-1}$. Here, $M_{\mathrm{s}}$ denotes the saturation magnetization, $\gamma=2.21 \times 10^{5} \mathrm{~mA}^{-1} \mathrm{~s}^{-1}$ is related to the gyromagnetic factor, $\alpha$ is the damping constant which is generally considered in the range of $10^{-3} \div 10^{-2}, \beta$ is the dimensionless function describing the intensity of spintransfer torque, while $\mathbf{p}$ is the direction of spin polarization.

In our analysis of uniaxial magnetic objects, the effective field is given by the following expression:

$$
\mathbf{h}_{\text {eff }}=D_{x} m_{x} \mathbf{e}_{x}-h_{a x} \mathbf{e}_{x}+h_{a y} \mathbf{e}_{y}+h_{a z} \mathbf{e}_{z} .
$$

The coefficient $D_{x}$ accounts for the magnetocrystalline anisotropy and the demagnetizing field, while $h_{a x}, h_{a y}$ and $h_{a z}$ are the components of the normalized applied field $\mathbf{h}_{a}$, which is considered constant. Let us assume the spin polarization of the current is along the anisotropy axis $(\mathbf{p}=$ $\mathbf{e}_{x}$ ), while $\beta$ is constant, estimated to be smaller than $10^{-2}$. The assumption of constant $\beta$ is reasonable for small values of spin polarization $(\mathrm{P}<0.1)$, but it has to be viewed as a first approximation when larger values of $\mathrm{P}$ are considered. Due to the rotational symmetry of the problem around the $x$ axis, one can choose $y$ axis along the field projection on $(y$, $z$ ) plane. By simply renormalizing the applied field, $\beta$ parameter, and time $t$, constant $D_{x}$ can be removed from the expression of the applied field. Taking into account these observations and redefining the coordinate system in $y-z$ plane $\left(\mathbf{e}_{y}, \mathbf{e}_{z}\right)$, the effective field can be rewritten as follows:

$$
\mathbf{h}_{e f f}=m_{x} \mathbf{e}_{x}-h_{x} \mathbf{e}_{x}+h_{y} \mathbf{e}_{y} .
$$

The resulting configuration is represented in Figure 1.

It is well known that the quasi-static critical field curve in the absence of spin-polarized current (i.e. $\beta=0$ ) is given by the Stoner-Wohlfarth (SW) astroid. The presence of a spinpolarized current is expected to have a strong influence on this loss-of-metastability curve, as well as on the general structural stability of the magnetization dynamics. A first step in the quasi-static analysis of our system is to find the fixed points of dynamics (1) with the effective field defined by (3). The fixed points, which will be denoted by $\mathbf{m}_{0}$, represent the metastable magnetic states supported by the magnetic field and the spin-polarized current. They are characterized by the time derivative of $\mathbf{m}_{0}$ being zero at any instant of time and by $\left\|\mathbf{m}_{0}(0)\right\|=1$, which is equivalent to $\mathbf{h}_{\text {eff }}\left(\mathbf{m}_{0}\right)-\beta \mathbf{m}_{0} \times \mathbf{e}_{x}=\lambda \mathbf{m}_{0}$ and $\left\|\mathbf{m}_{0}(0)\right\|=1$. The parameter $\lambda$ is to be determined, along with the three components of $\mathbf{m}_{0}$, from the previous relations, that can be written as:

$$
\left\{\begin{array}{l}
(1-\lambda) m_{0 x}=h_{x} \\
\lambda m_{0 y}+\beta m_{0 z}=h_{y} \\
-\beta m_{0 y}+\lambda m_{0 z}=0
\end{array},\right.
$$

$$
m_{0 x}^{2}+m_{0 y}^{2}+m_{0 z}^{2}=1
$$


Except the singular cases when $h_{x}$ is zero or $\lambda$ is 1 , linear system (4) has a unique solution:

$m_{0 x}=\frac{h \cos \varphi}{(1-\lambda)}, \quad m_{0 y}=\frac{\lambda h \sin \varphi}{\lambda^{2}+\beta^{2}}, \quad m_{0 z}=\frac{\beta h \sin \varphi}{\lambda^{2}+\beta^{2}}$,

where $\varphi$ is the angle between the magnetic field and the anisotropy axis, while $h$ is the magnitude of the applied field. In order to achieve norm condition (5), parameter $\lambda$ has to satisfy the following relation:

$$
\frac{\cos ^{2} \varphi}{(1-\lambda)^{2}}+\frac{\sin ^{2} \varphi}{\lambda^{2}+\beta^{2}}=\frac{1}{h^{2}} \text {. }
$$

Let us denote the expression from the left-hand side of equation (7) by $\mathrm{F}^{\beta}(\lambda)$ and plot its graph with respect to $\beta$ (see Figure 2). The solutions for equation (7) can be geometrically interpreted as the intersection of this graph with the line parallel to the $\lambda$-axis drawn at height $1 / \mathrm{h}^{2}$. It is apparent that the magnitude of the applied field does not affect the graph of $F^{\beta}(\lambda)$. Consequently, the solutions corresponding to different magnitudes of a magnetic field applied along a fixed direction are obtained by simply translating the parallel to $\lambda$-axis.

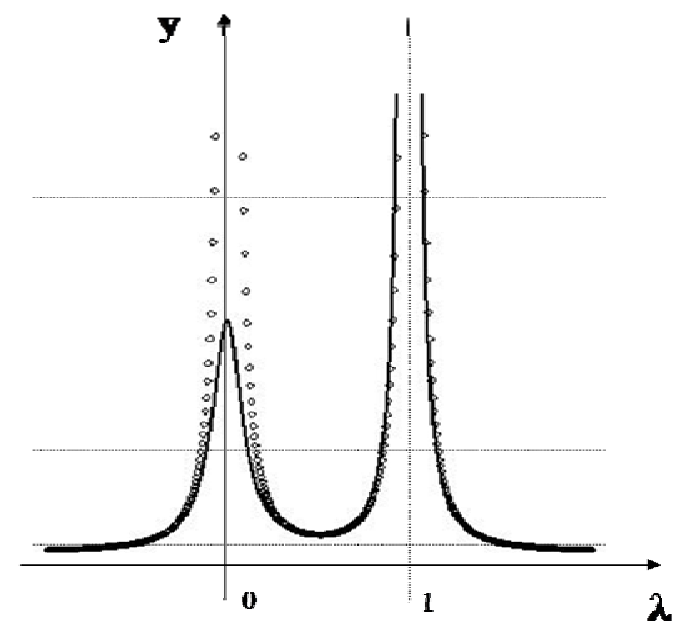

Figure 2. The graph of function $\mathrm{F}^{\beta}$ for a typical non-zero value of small parameter $\beta$ (represented by continuous line) and for limit case $\beta=0$ (represented by symbols). The pointed lines parallel to $\lambda$ - axis illustrate three representative cases for equation (7).

By taking into account the small value of parameter $\beta$, which is in the order of $10^{-3} \div 10^{-2}$, several conclusions can be drawn with respect to function $\mathrm{F}^{\beta}$. This function has a singular point in $\lambda=1$, a maximum point (denoted by $\lambda_{1}$ ) close to 0 , and a minimum point (denoted by $\lambda_{2}$ ) close to a minimum point for $\mathrm{F}^{0}$. Moreover, the (local maximum) value of function $\mathrm{F}^{\beta}$ in $\lambda_{1}$ can be approximated by the following expression:

$$
F^{\beta}\left(\lambda_{1}\right) \approx \frac{\sin ^{2} \varphi}{\beta^{2}}+\cos ^{2} \varphi
$$

and the minimum point $\lambda_{2}$ can be approximated by the solution of the following equation:

$$
\frac{\partial F^{0}}{\partial \lambda}=\frac{2 \cos ^{2} \varphi}{(1-\lambda)^{3}}-\frac{2 \sin ^{2} \varphi}{\lambda^{3}}=0 .
$$

Consequently, it leads to the following expression for $\lambda_{2}$ :

$$
\lambda_{2} \approx \frac{\tan ^{2 / 3} \varphi}{1+\tan ^{2 / 3} \varphi}
$$

and the following approximate value for function $\mathrm{F}^{\beta}$ :

$$
F^{\beta}\left(\lambda_{2}\right)=\left(\cos ^{2 / 3} \varphi+\sin ^{2 / 3} \varphi\right)^{3} .
$$

Taking into account relation (7), and the local extrema values (8) and (11), two important values for the magnitude of the applied field can be computed:

$$
\begin{aligned}
& h_{1}=\frac{\beta}{\sqrt{\beta^{2} \cos ^{2} \varphi+\sin ^{2} \varphi}}, \\
& h_{2}=\left(\cos ^{2 / 3} \varphi+\sin ^{2 / 3} \varphi\right)^{-3 / 2} .
\end{aligned}
$$

The last expression can be easily recognized as the Stoner-Wohlfarth astroid, which represents the critical field curve in the absence of the spin polarized current.

As a result, our analysis of equation (7) can be summarized as follows:

- For $h \in\left(0, h_{1}\right)$, equation (7) has two solutions, one larger than 1 , the other in between $\lambda_{2}$ and 1 ;

- For $h \in\left(h_{1}, h_{2}\right)$, equation (7) has four solutions, one larger than 1 , another in between $\lambda_{2}$ and 1 , a third one in between $\lambda_{1}$ (approximately zero) and $\lambda_{2}$, and the last one smaller than $\lambda_{1}$;

- For $h>h_{2}$, equation (7) has two solutions, one larger than 1 and the other smaller than $\lambda_{1}$.

Since equation (7) is quartic with respect to $\lambda$, explicit forms for the solutions are readily available. Due to the limitations related to the article length, we do not present these expressions here, but the reader can derive them by using the general solution of the quartic equation. The values for the magnetization components $\mathrm{m}_{0 \mathrm{x}}, \mathrm{m}_{0 \mathrm{y}}$ and $\mathrm{m}_{0 \mathrm{z}}$ corresponding to the fixed points of LLGS dynamics can be explicitly found by plugging the expressions obtained for $\lambda$ in formula (6).

In order to analyze the stability of these fixed points, let us consider small perturbations $\delta \mathbf{m},\|\delta \mathbf{m}\|<<1$. Since the magnetization norm is conserved along the trajectories of (1), the small perturbation has to satisfy the relation $\mathbf{m}_{0} \bullet \delta \mathbf{m}=0$, which means that the perturbation takes place in the plane orthogonal to fixed point $\mathbf{m}_{0}$. By plugging $\mathbf{m}_{0}+\delta \mathbf{m}$ in equation (1), where the effective field is given by (3), and by taking into account only first order terms in $\delta \mathrm{m}$, one can derive a linear differential system for the components of $\delta \mathbf{m}$ found in the plane perpendicular to $\mathbf{m}_{0}$. The determinant and trace for matrix A of this linear differential system controls the stability of fixed point $\mathbf{m}_{0}$. As a result of these calculations, the following expressions for the matrix determinant and trace are found:

$$
\begin{aligned}
& \operatorname{det} A=\frac{1}{1+\alpha^{2}}\left[\lambda_{0}^{2}-\frac{h^{2} \sin ^{2} \varphi}{\lambda_{0}}+\frac{\beta^{2} h^{2} \cos ^{2} \varphi}{\left(1-\lambda_{0}\right)^{2}}\right], \\
& \operatorname{tr} A=\frac{-2 \alpha}{1+\alpha^{2}}\left[\lambda_{0}-\frac{h^{2} \sin ^{2} \varphi}{2 \lambda_{0}^{2}}+\frac{\beta}{\alpha} \frac{h \cos \varphi}{1-\lambda_{0}}\right] .
\end{aligned}
$$

Although the complete analysis of all fixed points' stability is now possible, we limit here our discussion to the 
deviations from the $\mathrm{SW}$ astroid produced by the presence of spin-polarized current. For this purpose, we need to find the stable fixed points in the absence of the external magnetic field and to follow the stability of the fixed points continuously connected to "zero" fixed points when the magnetic field is "slowly" changed. The critical field curve or loss-of-metastability field curve is obtained by considering, for each direction in $\mathrm{x}-\mathrm{y}$ plane, the field at which the stability is lost. Let us mention that "slowly" actually means slower than the magnetization relaxation time needed to adjust to the new external conditions, which is on the order of nanoseconds.

In the absence of the external magnetic field $(\mathrm{h}=0)$, a different analysis is needed since our derivation has essentially used $h_{x} \neq 0$ starting from equation (6). When $h=0$ the last two equations from (4) imply that $\mathrm{m}_{0 \mathrm{y}}=0$ and $\mathrm{m}_{0 \mathrm{z}}=0$, and consequently, $\mathrm{m}_{0 \mathrm{x}}$ can only be +1 or -1 , according to relation (5). For the stability analysis of fixed points $\left( \pm \mathbf{e}_{x}\right)$, the perturbation technique leads to the following expressions for the determinant and trace of the matrix corresponding linear differential systems:

$$
\operatorname{det} A=\frac{1}{1+\alpha^{2}}\left(1+\beta^{2}\right), \quad \operatorname{tr} A=-\frac{2}{1+\alpha^{2}}(\alpha \mp \beta) .
$$

It is apparent from (16) that the $\operatorname{det} \mathrm{A}>0$, which means that both fixed points $\left( \pm \mathbf{e}_{x}\right)$ are foci, while their stability is given by the sign of the trace. In conclusion, $+\mathbf{e}_{\mathrm{x}}$ and $-\mathbf{e}_{\mathrm{x}}$ are stable focus when $\beta<\alpha$ and $\beta>-\alpha$ respectively.

Now we can proceed to the second step in our derivation, namely to follow the stability of the fixed points continuously connected to "zero" fixed points when the magnetic field is "slowly" changed. Let us consider the magnetization in equilibrium position $\mathrm{m}=\mathrm{ex}$ and a spinpolarized current with $\beta<\alpha$. According to the previous conclusions, position $\mathbf{m}=\mathbf{e}_{\mathrm{x}}$ continues to be a stable fixed point under the latter conditions as well. Now, let us apply an external magnetic field in a direction oriented at an obtuse angle $(\pi-\varphi)$ with $\mathbf{e}_{\mathrm{x}}$. This field is increased at a relatively small pace in order to allow the magnetization to settle in a stable fix point or a limit cycle (as it is mentioned above, this process is actually a relatively fast one which takes place on the order of nanoseconds). It is clear from Figure 2 that the magnetization will change continuously, according to the solution of equation (7) belonging to interval $\left(\lambda_{2}, 1\right)$, until the corresponding fixed point losses either its fixed point property (i.e. $h$ reaches $h_{2}$ ) or its stability. In the absence of the spin polarized current, the first scenario is valid and it leads to the following expression of the critical field:

$$
h_{c r}=\left(\cos ^{2 / 3} \varphi+\sin ^{2 / 3} \varphi\right)^{-3 / 2},
$$

which is well-known from the Stoner and Wohlfarth analysis. In the presence of the spin polarized current, the second scenario is valid, as will be proven next. As one can observe from equation (7) and Figure 2, the solution of equation (7) belonging to interval $\left(\lambda_{2}, 1\right)$ is not essentially affected by the presence of spin polarized current since $\beta<<$ 1. Moreover, the positiveness of the determinant is actually improved by a non-zero $\beta$ according to formula (14). Nevertheless, the spin polarized current has a strong influence on the fixed point stability through the trace of the perturbation system matrix. The third term in formula (15), directly related to the spin polarized current, is proportional to ratio $\beta / \alpha$ and leads to zero value for the matrix trace for $h$ smaller than $\mathrm{h}_{2}$. From condition $\operatorname{tr} \mathrm{A}=0$, the implicit algebraic formula for the critical field in the presence of the spin polarized current is obtained:

$$
\lambda_{0}(h)-\frac{h^{2} \sin ^{2} \varphi}{2\left[\lambda_{0}(h)\right]^{2}}+\frac{\beta}{\alpha} \frac{h \cos \varphi}{1-\lambda_{0}(h)}=0,
$$

where $\lambda_{0}(\mathrm{~h})$ is the solution of quartic equation (7) belonging to interval $\left(\lambda_{2}, 1\right)$. By solving this equation for every value of angle $\varphi$ in $\left[0,90^{\circ}\right]$, the critical field curve (that can be coined as the generalized Stoner-Wohlfarth astroid) is obtained for the case of a nano-objects with uniaxial anisotropy subject to a spin polarized current characterized by parameter $\beta$. The results of these calculations are plotted in Figure 3 for several values of ratio $\beta / \alpha$.

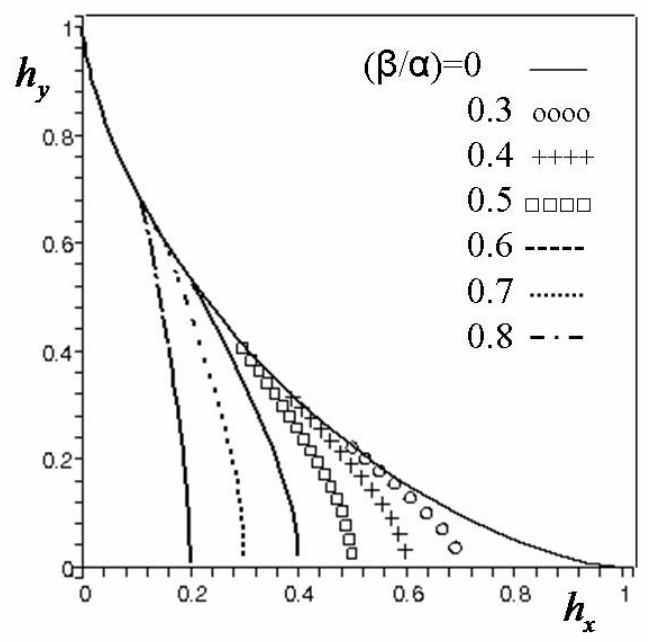

Figure 3. Critical magnetic field curve for spin polarized current assisted magnetization reversals in magnetic nano-objects plotted for several values of ratio $\beta / \alpha$.

As it is apparent from formula (18) coupled to (7), parameter $\beta$ is involved in the expression of critical field only with ratio $\beta / \alpha$, where $\alpha$ is the damping parameter. Let us remember that the classical Stoner-Wohlfarth astroid was derived based on free energy considerations independent of the magnetization dynamics. The generalized SW astroid (18) includes the damping parameter, so the magnetization dynamics is important for these quasi-static considerations. That is somehow expected from energy considerations since $\alpha$ determines the rate of energy dissipation, while $\beta$ is related to the energy added to the system by the interaction with the spin-polarized current. The relation between the energy added and the one dissipated by the system should be reflected in the limit-of-metastability field curve and that is taking into account in formula (18) by ratio $\beta / \alpha$. Another important difference between the generalized SW astroid and the classical one is related to the switching property. In the SW case, the astroid represents not only the limit-ofmetastability curve but also the switching curve, since the magnetization projection on the easy axis changes its sign when the magnetization jumps from the fixed point that losses its stability to the stable point. In the spin-polarized case, the generalized SW astroid represents the limit-of- 
metastability curve, but it does not always guarantee the magnetization switching. For example, when the energy added in the system compensates the energy dissipation, the magnetization might jump into a metastable oscillatory state, corresponding to a limit cycle in the structural stability analysis of LLGS. Such phenomena are now under intense research focus since they are expected to contribute to the development of high frequency radiation sources.

In the particular case when the external field is applied along the anisotropy axis (opposite to the magnetization orientation) equations (7) and (18) take the following forms:

$$
\frac{1}{\left[1-\lambda_{0}(h)\right]^{2}}=\frac{1}{h^{2}} \quad \text { and } \quad \lambda_{0}(h)-\frac{\beta}{\alpha} \frac{h}{1-\lambda_{0}(h)}=0,
$$

which lead to the following formula for the critical field corresponding to the easy-axis orientation:

$$
h_{c r}=1-\frac{\beta}{\alpha} \text {. }
$$

Let us notice that the critical field for the easy axis is linearly dependent on ratio $\beta / \alpha$ and it is smaller than SW critical field by that ratio. An important observation related to this particular case is that the magnetization dynamics can be fully resolved due to the rotational symmetry of the problem. The proof of this observation and the explicit expressions for the magnetization dynamics will be presented elsewhere.

A final remark will be made at this time to address the influence of the spin polarized current of the precessional switching of magnetization [13-14]. First, let us mention that equation (1) is equivalent to the Landau-LifshitzSlonczewski equation:

$$
\frac{d \mathbf{m}}{d \tau}=-\mathbf{m} \times\left(\mathbf{h}_{e f f}-\beta \alpha \mathbf{e}_{x}\right)-\alpha \mathbf{m} \times \mathbf{m} \times\left(\mathbf{h}_{e f f}-\frac{\beta}{\alpha} \mathbf{e}_{x}\right),
$$

where $\tau=t /\left(1-\alpha^{2}\right)$, while the effective field is given by formula (3). It is apparent that the spin-polarized current has a much stronger influence on the "damping" term (the second term on the right hand side of (21)) than on the "precessional" term (the first term on the right hand side of (21)). Since both $\beta$ and $\alpha$ are small parameters, the contribution from the product $\beta \cdot \alpha$ can be completely ignored. Although the influence of the spin polarized current is extremely weak during the precessional part of the switching, it plays an important role on the final relaxation part, when the magnetization destination is actually determined. A complete analysis of precessional switching in the presence of spin-polarized current is under development in our group.

\section{ACKNOWLEDGMENTS}

This work has been supported by the Romanian Executive Agency for Higher Education and Research Funding, PN II Human Resources, contract number 13/1.10.2007. Anca Gîndulescu thanks European Union contract no. POSDRU/6/1.5/S/22 and Cyril Acholo thanks U.S. National Science Foundation, Partnership for Education and Research Materials, for their financial supports.

\section{REFERENCES}

[1] K. Stoev, F. Liu, Y. Chen, X. et al., "Demonstration and characterization of $130 \mathrm{~Gb} / \mathrm{in}^{2}$ magnetic recording systems," Journal of Applied Physics 93 (10), 6552 (2003)

[2] Z. Zhang, Y. C. Feng, T. Clinton, et al, "Magnetic recording demonstration over $100 \mathrm{~Gb} / \mathrm{in}^{2}$," IEEE Transactions on Magnetics 38 (5), 1861 (2002)

[3] M. Kryder and R. Gustafson, "High-density perpendicular recording -advances, issues, and extensibility," Journal of Magnetism and Magnetic Materials 287, 449, (2005)

[4] Hitachi Global Storage Technologies, News Release, 15 October 2007. http://www.hitachi.com/New/cnews/071015a.html

[5] Z. Gai, J.Y. Howe, J. Guo, D.A. Blom, et al., "Self-assembled FePt nanodot arrays with mono-dispersion and -orientation," Applied Physics Letters 86, 023107 (2005)

[6] S. Sun, C.B. Murray, D. Weller, et al., "Monodisperse FePt Nanoparticles and Ferromagnetic FePt Nanocrystal Superlattices," Science 287, 1989 (2000)

[7] B. Engel, J. Akerman, B. Butcher, et al., "A 4-Mb toggle MRAM based on a novel bit and switching method," IEEE Transactions on Magnetics 41 (1), 132 (2005)

[8] R.P. Cowburn, "The future of universal memory," Materials Today 6 (7-8), 32 (2003).

[9] D. Weller and A. Moller, "Thermal effect limits in ultrahigh-density magnetic recording," IEEE Transactions on Magnetics 35 (6), 4423 (1999)

[10] J.G. Zhu, "New heights for hard disk drives," Materials Today 6 (78), 23 (2003)

[11] R.H. Koch, J.A. Katine, J.Z. Sun, "Time-Resolved Reversal of SpinTransfer Switching in a Nanomagnet," Physical Review Letters 92 (8), 088302 (2004)

[12] Y.B. Bazaliy, B.A. Jones, S. Zhang, "Current-induced magnetization switching in small domains of different anisotropies" Physical Review $B$ 69, 094421 (2004)

[13] M. Dimian, I. Mayergoyz, G. Bertotti, C. Serpico, "Multiscale analysis of magnetization dynamics driven by external fields," Journal of Applied Physics 99 (8), 08 G104 (2006)

[14] I. Mayergoyz, M. Dimian, G. Bertotti, Serpico, "Critical fields and pulse durations for precessional switching of perpendicular media," Journal of Applied Physics 97 (10), 10E509 (2005)

[15] D.C. Worledge, "Single-domain model for toggle MRAM," IBM Journal of Research \& Development 50 (1), 69 (2006)

[16] D. Cimpoesu, A. Stancu, L. Spinu, "Dynamic and temperature effects in toggle magnetic random access memory," Journal of Applied Physics 102 (1), 013915 (2007)

[17] H. Gavrila, "Heat-assisted magnetic recording," Journal of Optoelectronics and Advanced Materials 10 (7), 1796 (2008)

[18] R. Rottmayer, S. Batra, D. Buechel, et al., "Heat-Assisted Magnetic Recording," IEEE Transactions on Magnetics 42 (10), 2417 (2006)

[19] J. Slonczewski, "Current-driven excitation of magnetic multilayers," Journal of Magnetism and Magnetic Materials 159, L1 (1996)

[20] L. Berger, "Emission of spin waves by a magnetic multilayer traversed by a current," Physical Review B 54 (15), 9353 (1996)

[21] W. Weber, S. Riesen, H. C. Siegmann, "Magnetization Precession by Hot Spin Injection," Science 291, 1015 (2001)

[22] Y. Huai, F. Albert, P. Nguyen, et al., "Observation of spin-transfer switching in deep submicron-sized and low-resistance magnetic tunnel junctions," Applied Physics Letters 84, 3118 (2004)

[23] S. I. Kiselev, J. C. Sankey, I. W. Krivorotov, et al., "Microwave oscillations of a nanomagnet driven by a spin-polarized current," Nature 425 (6956), 380 (2003)

[24] B. Hillebrands, A. Thiaville (eds.), Spin dynamics in confined magnetic structures III, Springer (2006)

[25] S. A. Wolf, D. D. Awschalom, R.A. Buhrman, et al., "Spintronics: A Spin-Based Electronics Vision for the Future." Science, 294 (5546), 1488 (2001)

[26] A. Slavin, V. Tiberkevich, "Spin Wave Mode Excited by SpinPolarized Current in a Magnetic Nanocontact is a Standing SelfLocalized Wave Bullet," Physical Review Letters 95, 237201 (2005)

[27] J.Z. Sun, "Spin-current interaction with a monodomain magnetic body: A model study," Physical Review B 62, 570 (2000)

[28] G. Bertotti, A. Magni, R. Bonin, et al., "Bifurcation analysis of magnetization dynamics driven by spin transfer," Journal of Magnetism and Magnetic Materials 290-291, 522 (2005) 